\title{
Simulation Tools for Inclusive Design Solutions
}

\author{
Sujithra RAVISELVAM ${ }^{\mathrm{a}, 1}$, Shiroq AL-MEGREN ${ }^{\mathrm{b}}, \mathrm{Kyll}_{\mathrm{K}} \mathrm{KEANE}^{\mathrm{b}}$, \\ Katja HÖLTTÄ-OTTO ${ }^{c}$, Kristin L. WOOD a and Maria C. YANG ${ }^{\text {b }}$ \\ ${ }^{a}$ Engineering Product Development, Singapore University of Technology and Design, \\ Singapore \\ ${ }^{\mathrm{b}}$ Massachusetts Institute of Technology, United States \\ ${ }^{\mathrm{c}}$ Aalto University, Finland
}

\begin{abstract}
Disability has been redefined by the World Health Organization as a function of a person's interaction with the environment and not merely an innate part of a person. This redefinition highlights the need for inclusiveness in design solutions. To aid this, we apply and test the potential of different tools that restrict designers' physical abilities at deriving inclusive design perspectives among designers. Various tools and simulated conditions are often adopted in user-centered design to sup-port need-finding by eliciting rich data on users' needs and guide designers to empathize with users. Simulation tools that restrict designers' physical abilities have been applied to understand certain perspectives of people with physical challenges, yet these tools lack the ability to evoke an inclusive design perspective among designers. Through a co-creation workshop, participants were exposed to two forms of simulations: direct and situational physical impairments. This was achieved using different tools that simulate the same physical restriction. In this study, a noisecanceller and earphones were used to simulate a reduced hearing attention. Participants were asked to generate user needs and design functions by applying both the simulation tools. The study resultscomprise the outcomes of 33 participants who volunteered to participate in a co-design workshop that provided a venue for them to interact and work alongside users with physical challenges. This paper analyses the inclusiveness attained through different types of simulated conditions. With a growing need to create tools and technologies that delight the user, it is necessary to equip designers with the tools that would help them with the process. The study demonstrates the application and impact of one such tool.
\end{abstract}

Keywords. Simulation tools, creativity, inclusion, hearing impairment, assistive technology.

\section{Introduction}

Definitions for both Universal Design and Inclusive Design insists on accommodating the design needs experienced by a diverse group of users [1], [2]. Awareness of inclusive design practices has encouraged designers to explore various ways to understand the

\footnotetext{
1 Corresponding Author, Sujithra Raviselvam, Engineering Product Development, Singapore University of Technology and Design, 8 Somapah Rd, Singapore 487372; E-mail: sujithra_raviselvam@mymail.sutd.edu.sg.
} 
perspectives of such diverse user groups - particularly the elderly and users who experience physical challenges [3], [4]. Simulating a type of physical impairment, to an extent, has enabled designers to understand the perspectives of users with physical impairments. The Third Age Suit, developed in 1990 by the Ford Motor Company [5], was one of the initial simulation tools developed to offer engineers a deeper understanding of elderly users' needs while driving. Few other simulations suits include several generations of AGNES (Age Gain Now Empathy System) age simulation suits developed by the AgeLab at MIT [6] and the GERT suit by Produkt + Projekt Design team [7].

The use of simulation tools such as AGNES and GERT have proven effective by design practitioners [3], [8] and as an educational approach to teaching design students about need-finding [9]. Nevertheless, disability simulations have been criticized due to their focus on what it would be like to newly acquire a disability without accounting for coping mechanisms learned through life experiences [10]-[13], [8], [14]. A meta-analysis was carried out to evaluate the impact of ten studies that assessed attitude change when simulating cognitive, visual, hearing, and orthopaedic impairments [15]. The review findings show that there were only small attitude changes, and, in fact, in some, the change was for the worse. Another review of ten studies that simulate visual and auditory hallucinations found participants displaying negative emotions and physical distress [16]. In spite of the speculations on the application of simulation tools in impacting the attitude towards people with physical challenges, they have proven to be impactful at evoking empathy [13] and creativity among design practitioners [17]-[19].

In addition, inclusive design studies and products support that designs that address the needs experienced by users with physical challenges, in a way address the needs that are latent among the general population users. Products such as OXO GoodGrips houseware, typewriters, Folks kitchenware for blind [20], [21], StickEar [22] and GrOpener [23] are some examples of creative design solutions that were inspired from the needs experienced by people with physical challenges. Building on this, our previous work simulated different types of physical challenges to test its effect on participants' ability to apply them to identify latent user needs in medical device design[24], [25]. The aim was to build medical devices that reduced physical demands when necessary and enable inclusive interfaces. Although the simulated scenarios were effective at enabling the study participants to identify latent design needs, the participants seldom viewed them as an inclusive design solution. Majority of the needs were conceptualized as a need that would specifically assist people with physical challenges and not as a need that could enable inclusive user interaction. For example, with a blindfold (simulating reduced visual attention) as the applied simulation tool, the participants frequently quoted '...to help users with visual challenges.'

To explore the possibilities of applying simulation tools to evoke design solutions that are more inclusive, this work studied the impact of simulating situational physical impairments and its impact on design outcomes. Situational impairments have always been insisted as a secondary reason to have more inclusive design solutions. This refersto physical challenges that are experienced due to a particular context. For example, a mother carrying a child is a single-handed user during that particular situation [26]. Yet, simulating such situational disabilities is a less explored space for inclusive design ideation. We test the impact of simulating situational disabilities by comparing them against a simulated physical impairment while answering the following research questions. 
1) Does the design outcome differ between a simulated physical challenge and a simulated situational impairment?

2) Does the order and type of simulation impact the inclusiveness among the derived design concepts?

\section{Research Methodology}

This study was part of the Humanistic Co-design workshop that enabled the participants to work alongside users with physical challenges in Chennai, India. Thirty-three individuals ( 8 Female, 25 Male) participated in the study. Situational and direct simulation of hearing impairment was chosen as the case study. The situational hearing impairment, in this case, referred to a situation where a user has compromised hearing attention while listening to music on the earphones. To understand any potential order effects on the outcome, the participants were randomly assigned to two groups. Figure 1 illustrates the implemented research approach.

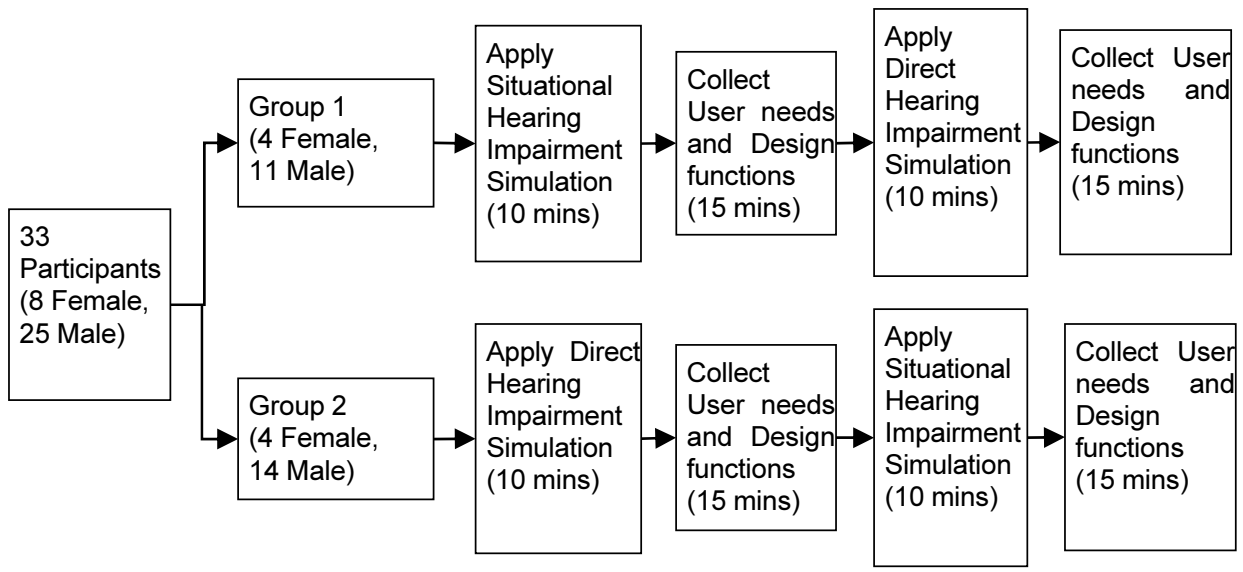

Figure 1. Research approach

The term 'Design functions' implied 'what must be done to the design' in order to achieve the identified user needs [27]. The study procedures met the criteria for exemption, where an Exemption Evaluation was submitted and approved by Massachusetts Institute of Technology's Committee on the Use of Humans as Experimental Subjects (COUHES). The situational and direct simulation of hearing impairment was achieved using two conditions: 1) Noise- canceller: direct hearing impairment simulation and 2) Earphones: situational hearing impairment simulation. The order in which the participants experienced the conditions differed based on their assigned group. As illustrated in Figure 1, participants from Group 1 experienced the condition with Earphones followed by the Noise-cancellers, and Group 2 experienced the condition with Noise-cancellers followed by the Earphones.

To test the given conditions, the participants were given a scenario and were asked to place themselves in the user's perspective and engage in the simulated scenario. The simulated scenarios placed the participants at a busy train station in Chennai, India, where they intend to board a train to a particular destination. Train tickets with four varied 
destinations (Gao, Delhi, Mumbai, and Bangalore) were distributed amongst the participants and platform numbers ( 1 to 4 ) were placed at various locations in the study room. The noise was introduced to the room via Bluetooth speakers to replicate the ambience of a noisy station. Additionally, a number of researchers were scattered amongst the participants and were asked to imitate strangers asking for directions to a certain platform or inquiring about a departure time for a particular train. Platform announcements were carried out audibly by one of the researchers and constituted the following: "The train heading to 'CITY' will be departing from platform 'PLATFORM NUMBER' in 'MINUTES' minutes."

In the Noise-canceller condition, the scenario was presented as follows:

"Your user is at a noisy train station in Chennai. There is an announcement being made about a rescheduled timing of their train to a particular destination. Your user is aperson with hearing impairment and may not be able to hear the announcement. How would you design an announcement system that addresses this situation?"

$3 \mathrm{M}$ noise-cancelling headgears with additional earplugs were distributed among the participants. After reading the scenario, the participants were asked to put on the earplugs and the noise cancelling headgear to simulate hearing impairment. The simulated scenario as described above commenced as soon as all participants were in their simulation for direct hearing impairment gear. Figure 2 shares a scenario from the Noisecanceller condition.

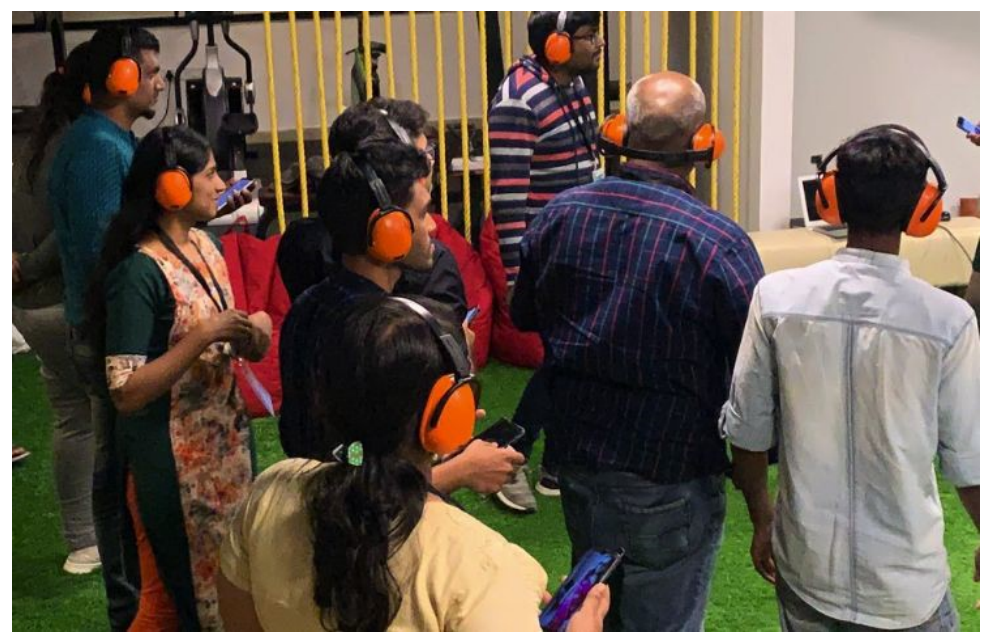

Figure 2. Participants experiencing the noise-canceller condition using a noise-cancelling headgear.

In the Earphone condition, the scenario was presented as follows:

"Your user is at a noisy train station in Chennai. There is an announcement being made after a rescheduled timing of their train to a particular destination. You user is listening to music using noise-cancelling headphones and may not be able to hear the announcement. How would you design an announcement system that addresses this situation?"

For this scenario, participants were asked to bring and use their own earphones. The simulated scenario commenced once the participants started to play music on their earphones. 
Following each scenario that lasted for 10 mins, using a google form, participants were given 15 minutes to record the user needs and design functions that are important to improve the design of the simulated announcement system. The identified design functions were used to evaluate the study outcomes.

\section{Results and Analysis}

The design functions were coded to categorize them based on the inclusiveness of the design functions shared by the participants. They were categorized into the categories:

Inclusive: The proposed design would benefit both users with hearing impairments and the rest of the general population users.

Assistive: The proposed design would benefit users with hearing impairments butwould not be preferred by the rest of the general population users.

Excluding: The proposed design would not benefit users with hearing impairments

Vague: Not a clear design description

Two researchers, one with an engineering design background and one with computer science and design background, rated a sample of the design functions shared by the participants based on the above-mentioned categories. Upon achieving 84.2\% similarity between the ratings provided by both the researchers, one researcher continued to bin the rest of the design functions. Table 1 lists the total number of design functions listed by the participants from both groups under each category.

Table 1. The number of design functions listed by the participants from Group 1 and Group 2.

\begin{tabular}{lcccc}
\hline & \multicolumn{2}{c}{ Group 1 $(\mathbf{n}=\mathbf{1 5})$} & \multicolumn{2}{c}{ Group 2 $(\mathbf{n = 1 8})$} \\
\hline Categories & Earphones & Noise-cancellers & Noise-cancellers & Earphones \\
Inclusive & 12 & 16 & 15 & $\mathbf{1 4}$ \\
Assistive & 0 & 1 & 12 & $\mathbf{4}$ \\
Excluding & 2 & 1 & 0 & $\mathbf{4}$ \\
Vague & 3 & 1 & 2 & $\mathbf{1}$ \\
\hline $\begin{array}{l}\text { Total number of } \\
\text { ideas }\end{array}$ & $\mathbf{1 7}$ & $\mathbf{1 9}$ & $\mathbf{2 9}$ & $\mathbf{2 3}$ \\
\hline
\end{tabular}

Results displayed in Table 1 shows that the Earphones condition was more impactful towards generating Inclusive design solutions among the Group 1 participants. To understand this further, Table 2 shares the percentage of 'Inclusive vs Only Inclusive', 'Assistive' vs 'Only Assistive' and 'Excluding' vs 'Only Excluding' design functions. While 'Inclusive' refers to the percentage of inclusive design functions identified by all participants, 'Only Inclusive' refers to the percentage of inclusive design functions listed by the participants whose 'Inclusive' category design function(s) were neither accompanied by 'Assistive' nor 'Excluding' category design functions. Similarly, 'Only Assistive' and 'Only Excluding' refers to design functions that were not paired with either of the other two categories. 
Table 2. Percentage of Inclusive, Assistive and Excluding design functions shared by the participants.

\begin{tabular}{lcccc}
\hline & \multicolumn{2}{c}{ Group 1 $(\mathbf{n}=\mathbf{1 5})$} & \multicolumn{2}{c}{ Group 2(n=18) } \\
\hline Categories & Earphones & Noise-cancellers & Noise-cancellers & Earphones \\
Inclusive & $86 \%$ & $86 \%$ & $61 \%$ & $67 \%$ \\
Only Inclusive & $64 \%$ & $78 \%$ & $44 \%$ & $61 \%$ \\
Assistive & $0 \%$ & $7 \%$ & $44 \%$ & $5 \%$ \\
Only Assistive & $0 \%$ & $0 \%$ & $28 \%$ & $5 \%$ \\
Excluding & $14 \%$ & $7 \%$ & $0 \%$ & $22 \%$ \\
Only Excluding & $7 \%$ & $7 \%$ & $0 \%$ & $22 \%$ \\
\hline
\end{tabular}

A comparison between Table 1 and Table 2 shows that although the Earphones condition encouraged more inclusive solutions among both the groups, this impact was more prominent among Group 1 participants who started with the Earphones (Situational impairment) condition. Table 3 lists some of the ideas listed by the participants based on their categories.

Table 3. Exemplar design functions shared by the participants.

\begin{tabular}{|c|c|}
\hline Categories & Design functions listed by the participants \\
\hline Inclusive & $\begin{array}{l}\text { - We could send a message to the passengersabout the updates. } \\
\text { - This would require a centralized displayboard. } \\
\text { - By Placing Digital display on the platform which will be } \\
\text { changed by getting anyresponse. }\end{array}$ \\
\hline Assistive & $\begin{array}{l}\text { - Adding sign language and making it visibleto everyone so } \\
\text { Hard of hearing can understand. } \\
\text { A hearing aid can be given at free of cost and it can be user- } \\
\text { friendly that is much more compatible and convenient. } \\
\text { - A function to identify if an announcement is being made } \\
\text { through a voice recognition system and alert the user. }\end{array}$ \\
\hline Excluding & $\begin{array}{l}\text { By voice repeat announcement again. We need to } \\
\text { design a noise filter. } \\
\text { - Automatically lower music volume. }\end{array}$ \\
\hline Vague & $\begin{array}{l}\text { - Should be easy to use even to kids.Getting persons } \\
\text { attention. } \\
\text { - Transfer of timely information. }\end{array}$ \\
\hline
\end{tabular}

\section{Discussion}

This study was structured with a goal to understand the impact of simulated situational impairments in generating inclusive design solutions. Two scenarios with compromised hearing abilities were simulated. While one condition directed the participants to improve the user experience of a person with hearing impairment, the other condition directed them to improve the user experience of a person listening to music over earphones. With this, we answer the two research questions introduced earlier as follows. 
1) Does the design outcome differ between a simulated physical challenge and a simulated situational impairment?

Results observed in Table 1 and Table 2 support that the design functions identified by the participants did get influenced by each simulated condition. One interesting aspect observed among the design functions is that a majority of the assistive design functions added an additional task to the end-user. For example, a wearable that captures the sound and converts it as information thatcan be perceived by the user with hearing impairment. Whereas a majority of the inclusive design functions did not impose any additional gadget or a wearable for the user. Although both the situations expected the participants to design for a situation with compromised hearing attention, situational impairments evoked a higher percentage of inclusive design suggestions and reduced the inclination to assistive solutions.

2) Does the order and type of simulation impact the inclusiveness among the derived design concepts?

This could be answered from Table 2 where the Participants from Group 1 who experienced the simulated situational hearing impairment (Earphones) followed by simulated hearing impairment (Noise-cancellers), continued to maintain a higher number of inclusive design functions even under a direct simulation of hearing impairment. In Group 2, while the percentage distribution was split between Inclusive and Assistive designs under the condition with Noise-cancellers, the percentage of Excluding design functions increased after experiencing the Earphones. This could be due to the possibility that the participants had already listed an inclusive or assistive design function during the previous stage and hence preferred to provide a solution more specific for the situational impairment situation. Based on the current outcomes, it would be preferable to experience a simulated situational impairment followed by direct simulation of a physical challenge. Further analysis is necessary to verify if this would be the preferable order to apply the simulations that result in more inclusive design outcomes.

\section{Limitations and Future Work}

This study is a preliminary work that explored the potential of applying a simulated situational impairment for inclusive design solutions. A small sample size that did not accommodate a rigorous statistical analysis was one of the primary limitations. Future works will focus on expanding the sample size to verify this outcome and study the impact of other potential external influencing factors, such as participant's previous experience, gender and other demographic contexts. Following that, other types of situational impairments for vision, dexterity and single-hand usage would be tested to verify if the study outcomes hold true for other types of physical challenges.

\section{Conclusion}

A comparison between situational and direct simulation of hearing impairment was executed. The situational and direct simulation of hearing impairment was explored with two conditions: noise-canceller and earphones. Participants were randomly assigned into two groups to examine any potential order effects on the outcome (group 1: headphones followed by noise-cancelling simulation; group 2: noise-cancelling followed by 
earphones). In either condition, participants were given a scenario, which was then followed by a form to collect participants perception of user needs and design functions that are desired to improve the design of the simulated announcement system. The findings support the potential of situational impairments in evoking more inclusivedesign outcomes and reduced the inclination to propose assistive solutions. This impact was more prominent for the first group (Group 1) that was exposed to the situational condition followed by the direct simulation. This experiment is one of the initial attempts to study if incorporating inclusive perspectives in simulated scenarios could evoke inclusive design solutions.

\section{Acknowledgements}

The authors deeply appreciate the support provided by the Humanistic Co-design team, MIT India, MIT Ideation lab and SUTD-MIT International Design Centre (IDC) (http://www.sutd.edu.sg/idc.aspx). Any opinions, findings, or recommendations are those of the authors and do not necessarily reflect the views of the sponsors or collaborators.

\section{References}

[1] W. F. E. Preiser and E. Ostroff, Universal Design Handbook. McGraw Hill Professional, 2001.

[2] "What is inclusive design?" [Online]. Available: http:/www.inclusivedesigntoolkit.com/whatis/ whatis.html. [Accessed: 24-Feb-2020].

[3] C. Cardoso and P. J. Clarkson, "Simulation in user-centred design: helping designers to empathise with atypical users," Journal of Engineering Design, vol. 23, no. 1, pp. 1-22, Jan. 2012, doi: 10.1080/ 09544821003742650.

[4] S. Raviselvam, K. L. Wood, K. Hölttä-Otto, V. Tam, and K. Nagarajan, "A Lead User Approach to Universal Design - Involving Older Adults in the Design Process," Stud Health Technol Inform, vol. 229, pp. 131-140, 2016.

[5] "Ford's Age Suit Lets You Experience Life As A 104 Year Old Man," Wonderful Engineering, 13-Feb2016. [Online]. Available: https://wonderfulengineering.com/fords-age-suit-lets-you-experience-life-as-a104-year-old-man/. [Accessed: 29-Apr-2019].

[6] “AGNES (Age Gain Now Empathy System) | MIT AgeLab." [Online]. Available: http://agelab.mit.edu/ agnes-age-gain-now-empathy-system. [Accessed: 29-Apr-2019].

[7] H. L. Groza, S. B. Sebesi, and D. S. Mandru, "Age Simulation Suits for Training, Research and Development," in International Conference on Advancements of Medicine and Health Care through Technology; 12th - 15th October 2016, Cluj-Napoca, Romania, Cham, 2017, pp. 77-80, doi: 10.1007/9783-319-52875-5_17.

[8] R. Kamikubo, K. Higuchi, R. Yonetani, H. Koike, and Y. Sato, "Exploring the Role of Tunnel Vision Simulation in the Design Cycle of Accessible Interfaces," in Proceedings of the Internet of Accessible Things on - W4A '18, Lyon, France, 2018, pp. 1-10, doi: 10.1145/3192714.3192822.

[9] J. Pivik, J. McComas, I. MaCfarlane, and M. Laflamme, "Using virtual reality to teach disability awareness," Journal of Educational Computing Research, vol. 26, no. 2, pp. 203-218, 2002.

[10] G. Kiger, "Disability Simulations: Logical, Methodological and Ethical Issues," Disability, Handicap \& Society, vol. 7, no. 1, pp. 71-78, Jan. 1992, doi: 10.1080/02674649266780061.

[11] S. French, "Simulation exercises in disability awareness training: A critique," Disability, Handicap \& Society, vol. 7, no. 3, pp. 257-266, 1992.

[12] C. M. Colwell, "Simulating disabilities as a tool for altering individual perceptions of working with children with special needs," International Journal of Music Education, vol. 31, no. 1, pp. 68-77, 2013.

[13] M. R. Nario-Redmond, D. Gospodinov, and A. Cobb, "Crip for a day: The unintended negative consequences of disability simulations.," Rehabilitation Psychology, vol. 62, no. 3, pp. 324-333, Aug. 2017, doi: 10.1037/rep0000127. 
[14] C. L. Bennett and D. K. Rosner, "The Promise of Empathy: Design, Disability, and Knowing the 'Other,"” in Proceedings of the 2019 CHI Conference on Human Factors in Computing Systems - CHI '19, Glasgow, Scotland Uk, 2019, pp. 1-13, doi: 10.1145/3290605.3300528.

[15] Meta-Analysis of Disability Simulation Research - Ashley Flower, Matthew K. Burns, Nicole A. Bottsford-Miller, 2007." [Online]. Available: https://journals.sagepub.com/doi/abs/10.1177/ 07419325070280020601. [Accessed: 24-Feb-2020].

[16] S. Ando, S. Clement, E. A. Barley, and G. Thornicroft, "The simulation of hallucinations to reduce the stigma of schizophrenia: a systematic review," Schizophrenia Research, vol. 133, no. 1-3, pp. 8-16, 2011.

[17] S. Raviselvam, K. Hölttä-Otto, and K. L. Wood, "User Extreme Conditions to Enhance Designer Empathy and Creativity: Applications Using Visual Impairment," presented at the ASME 2016 International Design Engineering Technical Conferences and Computers and Information in Engineering Conference, 2016, doi: 10.1115/DETC2016-59602.

[18] N. Genco, D. Johnson, K. Hölttä-Otto, and C. C. Seepersad, "A Study of the Effectiveness of Empathic Experience Design as a Creativity Technique,” 2011, pp. 131-139, doi: 10.1115/DETC2011-48256.

[19] J. Lin and C. C. Seepersad, "Empathic lead users: the effects of extraordinary user experiences on customer needs analysis and product redesign," in ASME 2007 International Design Engineering Technical Conferences and Computers and Information in Engineering Conference, 2007, pp. 289-296.

[20] D. A. McAdams and V. Kostovich, “A Framework and Representation for Universal Product Design,” p. $14,2011$.

[21] K. Holmes, "5 Ways Inclusion Fuels Innovation," Mismatch, 16-Sep-2018. [Online]. Available: https://mismatch.design/stories/2018/09/16/5-ways-inclusion-fuels-innovation/. [Accessed: 24-Feb-2020].

[22] K. P. Yeo, S. Nanayakkara, and S. Ransiri, "StickEar: making everyday objects respond to sound," in Proceedings of the 26th annual ACM symposium on User interface software and technology, St. Andrews, Scotland, United Kingdom, 2013, pp. 221-226, doi: 10.1145/2501988.2502019.

[23] S. Sheth, "A bottle opener for champions | Yanko Design." [Online]. Available: https://www.yankodesign.com/2017/04/04/a-bottle-opener-for-champions/. [Accessed: 24-Feb-2020].

[24] S. Raviselvam, D. Anderson, K. Hölttä-Otto, and K. L. Wood, "Systematic Framework to Apply Extraordinary User Perspective to Capture Latent Needs Among Ordinary Users," presented at the ASME 2018 International Design Engineering Technical Conferences and Computers and Information in Engineering Conference, 2018, doi: 10.1115/DETC2018-86263.

[25] S. Raviselvam, K. Subburaj, K. L. Wood, and K. Hölttä-Otto, “An Extreme User Approach to Identify Latent Needs: Adaptation and Application in Medical Device Design," in Volume 7: 31st International Conference on Design Theory and Methodology, Anaheim, California, USA, 2019, p. V007T06A011, doi: 10.1115/DETC2019-98266.

[26] G. Vanderheiden, "Fundamental principles and priority setting for universal usability," in Proceedings on the 2000 conference on Universal Usability, Arlington, Virginia, USA, 2000, pp. 32-37, doi: $10.1145 / 355460.355469$.

[27] K. Otto and K. Wood, Product Design: Techniques in Reverse Engineering, Systematic Design, and New Product Development. 2001. New York: Prentice-Hall. 